\title{
Electronic Chips as Indicators of Radiation Hazard for Biological Structures Caused by Heavy Particles in Space
}

\section{Mikhail Panasyuk*}

Skobeltsyn Institute of Nuclear Physics, Lomonosov Moscow State University, Russia

\section{Introduction}

As a rule fluxes of heavy high-energy charged particles (HZEparticles) - nuclei and ions in space are small as compared to the proton and electron fluxes and can't cause noticeable dose effects. But they are in the focus of studies as the basic element which cause socalled single events effects in microelectronics and local distortions in biological structures at the molecular level. Among the other radiation influence effects their role is of the highest immediate interest both from the point of spacecrafts' safety and for risk reduction during the long duration manned space missions (for instance, Moon- or Marsflights).

\section{Discussion}

Basically there are two dangerous radiation [1] sources for spacecrafts flying at low orbits. They are galactic cosmic rays (GCR) high-energy nuclei of different elements (from hundreds $\mathrm{MeV} /$ nucleon up to hundreds $\mathrm{GeV} /$ nucleon) coming to the near-Earth space from interstellar space, and the radiation belts $(\mathrm{RB})$ - charged particles captured by the magnetic field of the Earth. The latter produce the highest hazard for low-orbital spacecrafts. The point is that while the radiation belts are located at high altitudes, in South Atlantic region they "slack" above the Earth's surface due to anomalous structure of the Earth's magnetic field. In this region magnetic field of the Earth is tapped as compared to the other regions. Therefore, for instance, the International space station flying at the altitude of about $400 \mathrm{~km}$ crosses this dangerous region only during 2-3 circuits.

Nevertheless at these orbits radiation danger also exists - it is caused by high-energy charged particles - protons and nuclei of more heavy elements captured by the magnetic field of the Earth. Their energy reaches huge values (up to $1000 \mathrm{MeV}$ for protons) and spacecraft [2] envelope is not a barrier for them. These particles cause problems for on-board electronics. Currently electronics (chips) consists of high-density integration devices for with microscopic size clearences between current-conducting layers. Entering this structure highenergy charged particles disturb electro physical properties of chips and cause reversible or irreversible events in their operation. Collected sufficiently wide experimental material enables to make a categorical conclusion that South Atlantic region is the most hazardous for the low-orbit spacecrafts electronics. Mainly for different spacecrafts most of the events occur in this anomalous region. But such events also happen out of the anomaly, where they are produced by GCR influence. At low orbits events produces by GCR happen much more rarely because GCR flux is much lower than RB's one.

Is it possible to fight against this phenomenon? It is a burning problem which is far from being solved. The main difficulty is that we still do not know all the details of physical processes running inside such a complicated device as present-day microchip. In this case interaction of new technologies and natural phenomena produce new problems.
It is interesting that similar phenomena occur during interactions of heavy charged particles with biological structures in space: they are DNA disorder, neurones' damages and many other phenomena. They produce problems for long-duration manned space missions. GCR fluxes among which there are also heavy particles can cause a number of un diserable processes in biological structures including irreversible events. HZE-particles enable to cause changes in DNA structure. Under the influence of radiation water - the basic matter of biological tissues - becomes ionized, and it results in production of free radicals, which can destroy DNA molecular links. Scenarium of direct DNA damages during TZE-particles slowing down is also entirely possible. Some astronauts - veterans of long-duration space missions are addicted to serious chromosome aberration of blood cells which can bear witness of the beginning of cancerogenic lesions formation. Maybe these lesions will not cause cancer [3] if they are not prone to mutations, but apparently they are potentially dangerous. TZE-particles also influence on the human's eye causing damage of the eye's retina and lens.

It's necessary to note that there are a lot of disputable points in the mechanism of TZE-particles with cell structures and the problem within the frames of radiation biology is far from being solved. For instance, probability of the foregoing radiation disorder is not necessarily proportional to the total radiation dose. Different aberrations depend on the total dose in different ways: for some types of abberations small doses are necessary, while others need significantly higher doses. Synergetic effect of different types of radiation (for instance, TZEparticles influence compared with gamma-emission and electrons influence) is also not absolutely clear. Also the change of cognitive functions of the higher mammals after TZE-particles influence must be noted. Its reason is disorder at molecular level, which is not studied yet.

\section{Conclusions}

Without any doubt, TZE-particles influence on biological structures in space is a current problem both from the point of the studies of the nature of this phenomena, and for the safety of the longduration space missions for humans. At the same time, consistency of the results of the influence of TZE-component of space radiation [4] both on biological structures and on electronic chips suggests using of the latter as indicators of potential danger.

*Corresponding author: Mikhail Panasyuk, Skobeltsyn Institute of Nuclear Physics, Lomonosov Moscow State University, Russia, E-mail: panasyuk@sinp.msu.ru

Received October 31, 2013; Accepted November 19, 2013; Published November 21, 2013

Citation: Panasyuk M (2013) Electronic Chips as Indicators of Radiation Hazard for Biological Structures Caused by Heavy Particles in Space. Astrobiol Outreach 1: 106. doi: 10.4172/2332-2519.1000106

Copyright: (c) 2013 Panasyuk M. This is an open-access article distributed under the terms of the Creative Commons Attribution License, which permits unrestricted use, distribution, and reproduction in any medium, provided the original author and source are credited. 
Citation: Panasyuk M (2013) Electronic Chips as Indicators of Radiation Hazard for Biological Structures Caused by Heavy Particles in Space. Astrobiol Outreach 1: 106. doi: 10.4172/2332-2519.1000106

Page 2 of 2

\section{References}

1. Bashkirov V, Kuznetsov N, Nymmik R (1999) An analysis of the SEU rate of microcircuits exposed by the various components of space radiation. Radiation Measurements 30: 427-433.

2. Chechenin NG,Kadmenskii AG, Motaweh GH, Panasyuk M I(2012) Impact of High-Energy Cosmic-Ray Protons and lons on the Elements of Spacecraft
On-Board Devices. Journal of Surface Investigation. X ray, Synchrotron and Neutron Techniques 6: 303-313.

3. Cucinotta FA, Hu S, Shwadron NA( 2010) Space radiation Cancer Risk Projections and Uncertaities. NASA/TP-2011-216155.

4. Ma TP, Paul DV (1989) Ionization radiation effects in MOS devices and circuits, Wiley, USA. 